\title{
Experiencia en el manejo de heridas por mordedura de perro en un hospital de tercer nivel de cirugía plástica y reconstructiva en México
}

\author{
Experience in the management of acute wounds by dog bite in a hospital of third level \\ of plastic and reconstructive surgery in Mexico
}

\author{
Enrique Chávez-Serna*, Laura Andrade-Delgado, Rogelio Martínez-Wagner, Carlos Altamirano-Arcos, \\ Israel Espino-Gaucín y Luciano Nahas-Combina \\ Secretaría de Salud, División de Cirugía Plástica y Reconstructiva, Hospital General Dr. Manuel Gea González, Ciudad de México, México
}

\section{Resumen}

Antecedentes: Las heridas por mordedura de perro constituyen un problema de salud pública creciente que involucra a todos los niveles de atención. Pueden variar en gravedad, desde heridas superficiales (piel y tejido subcutáneo) hasta desvitalización y pérdida de tejidos, con presencia de infecciones, complicaciones y secuelas estéticas y funcionales que requieren tratamiento especializado. Objetivo: Describir la experiencia de 7 años en el manejo de heridas por mordedura de perro y proponer un algoritmo de manejo en un centro de referencia en cirugía plástica y reconstructiva en México. Método: Se realizó un estudio retrospectivo en el que se incluyeron todos los registros de pacientes con heridas por mordedura de perro entre julio de 2010 y agosto de 2017. Las variables recabadas fueron edad, sexo, localización anatómica, características de la herida y manejo terapéutico. Resultados: Se obtuvo un total de 416 pacientes. El 63\% de las lesiones ocurrieron en menores de 18 años. La mayoría de las heridas (88.2\%) fueron aisladas en una región anatómica. La localización más frecuente fue la cara (61.3\%). De todos los casos, el $74.3 \%$ se resolvieron solo con cierre primario, el $21.4 \%$ requirió además algún proceso reconstructivo, el $2.9 \%$ no requirió cierre y el $1.4 \%$ de los pacientes fueron hospitalizados. Todos los pacientes recibieron antibióticos profilácticos. El $2 \%$ reportaron infección posterior al manejo con cierre primario. Conclusiones: El correcto abordaje de las heridas por mordedura de perro en todos los niveles de atención mejora el pronóstico de los pacientes. Las heridas complejas deben ser manejadas inmediatamente en un centro de segundo o tercer nivel con cirugía plástica y reconstructiva. La mayoría de las heridas por mordedura de perro pueden ser reparadas con cierre primario y otras técnicas reconstructivas con seguridad.

PALABRAS CLAVE: Cierre primario. Cirugía reconstructiva. Herida quirúrgica. Heridas y lesiones. Manejo de heridas por mordedura. Mordedura de perro.

\section{Abstract}

Background: Dog bite injuries represent an important public health problem, involving all levels of care. They can vary in severity, from superficial wounds (skin and subcutaneous tissue) to devitalization and tissue loss, with the presence of systemic infections, aestethic and functional sequels that require specialized treatment. Objective: To describe and share the 7-year experience in the management of dog bite wounds in a plastic and reconstructive surgery referral center in Mexico. Method: A retrospective study was conducted where all patients with dog bite wounds that came to the plastic and reconstructive emergency

\author{
Correspondencia: \\ *Enrique Chávez-Serna \\ Calzada de Tlalpan, 4800 \\ Col. Belisario Domínguez, Sección XVI, Del. Tlalpan \\ C.P. 14080 , Ciudad de México, México \\ E-mail: enrique.chavez.serna@gmail.com
}

Fecha de recepción: 22-08-2018

Fecha de aceptación: 05-04-2019

DOI: 10.24875/CIRU.19000678
Cir Cir. 2019;87:528-539

Contents available at PubMed www.cirugiaycirujanos.com 
department were enrolled from July 2010 to August 2017. The collected patient demographic characteristics included age, age group, and sex. The collected injury variables included anatomical location, wound characteristics, antibiotic therapy and therapeutic management. Results: $A$ total of 416 patients were enrolled, $63 \%$ of the dog bites occurred in children under 18 years of age. The majority of the wounds were found in an isolated anatomical region $88.2 \%$. The most frequent location was the face in $61.3 \%$. Of all the cases $74.3 \%$ were treated only with primary wound closure, $21.4 \%$ also required some reconstructive surgical procedure, $2.9 \%$ did not require wound closure, and $1.4 \%$ were hospitalized. All patients received prophylactic antibiotics. $2 \%$ reported infection after the management with primary wound closure. Conclusions: The right management of dog bite wounds in all the health care levels improve the patient prognosis. Complex dog bite wounds must be treated immediately by the plastic and reconstructive surgery department in second or third levels of healthcare. Most of the wounds can be managed by primary closure and other reconstructive techniques with confidence.

KEY WORDS: Wounds. Injuries. Dog bite. Management bite wounds. Surgical wound. Wound closure. Reconstructive surgery.

\section{Introducción}

De acuerdo con la Sociedad Americana de Cirujanos Plásticos, 28,000 personas requieren cirugía reconstructiva anualmente por mordedura de perro ${ }^{1}$. En México, aunque el número de procedimientos quirúrgicos realizados por este problema es desconocido, en nuestra institución el número de pacientes que requieren manejo reconstructivo por la complejidad de las lesiones y hospitalización ha ido en aumento. A esto se añaden los elevados costos que conlleva el tratamiento inicial, y las secuelas y complicaciones que pueden derivar de la mordedura de perro. El objetivo de este estudio es conocer la epidemiología de este fenómeno en México, las características de las lesiones, la necesidad de realizar procedimientos reconstructivos en comparación con el cierre primario, describir la experiencia en el manejo en un centro de referencia de tercer nivel en cirugía plástica y reconstructiva, y finalmente establecer la propuesta de un algoritmo de tratamiento que optimice la atención en todos los eslabones de salud de este complejo grupo de pacientes.

\section{Método}

Se realizó un estudio retrospectivo en el Hospital General Dr. Manuel Gea González, considerado como centro de referencia de tercer nivel en cirugía plástica y reconstructiva en el sur de la Ciudad de México. Se incluyeron todos los registros electrónicos de pacientes que acudieron al servicio de urgencias de cirugía plástica y reconstructiva por presentar heridas por mordedura de perro que cumplieran con el seguimiento de consultas, registro fotográfico antes y después de la atención, y con apego al tratamiento, en el periodo de julio de 2010 a agosto de 2017. Todos los pacientes recibieron tratamiento quirúrgico estandarizado, que consistió en aplicación de anestesia local con lidocaína en el área afectada, posteriormente aseo quirúrgico con irrigación profusa con solución salina al $0.9 \%$ y clorhexidina por 15 minutos, seguido de desbridación de bordes no viables, exploración quirúrgica, cierre primario 0 procedimiento reconstructivo dependiendo de la gravedad y las características de la herida, y prescripción de antibióticos profilácticos. El comité interno de investigación y ética del hospital aprobó el estudio. Las variables demográficas recabadas fueron la edad y el sexo. Las variables relacionadas con la mordedura fueron características de la lesión, localización anatómica, antibioticoterapia y manejo empleado. Todos los pacientes firmaron el consentimiento informado para el uso de fotografías.

\section{Resultados}

Se obtuvieron 416 registros electrónicos de pacientes de la base de datos de urgencias del servicio de cirugía plástica y reconstructiva. La revisión de los registros arrojó que la edad promedio fue de 19.7 años (rango: 2 meses a 92 años), con una mediana de 11 años. De ellos, 240 eran hombres (57.7\%) y 176 eran mujeres (42.3\%). La incidencia más alta ocurrió en los menores de 18 años, con 262 casos (63\%) (Tabla 1). Se clasificaron los casos en cinco grupos de edad para facilitar el análisis (Fig. 1). De estos, los escolares (6-12 años) fueron el grupo más afectado, con 90 casos $(21.6 \%)$, seguidos de los preescolares (3-6 años) con 89 casos (21.4\%), los lactantes (1 mes a 2 años y 11 meses) con 43 casos (10.3\%) y los adolescentes (13-18 años) con 40 casos (9.6\%); los menos afectados fueron la población menor de 18 años. Para los mayores de 18 años se registraron 154 casos $(37 \%)$ y la incidencia más alta en este grupo 
Tabla 1. Características de las heridas en cierre primario vs. procesos reconstructivos

\begin{tabular}{|c|c|c|c|c|}
\hline & Cierre primario & Procesos reconstructivos & Total & $\%$ \\
\hline \multicolumn{5}{|l|}{ Localización anatómica } \\
\hline - Cara & 201 & 49 & 250 & 63.1 \\
\hline - Cabeza y cuello & 6 & 0 & 6 & 1.5 \\
\hline - Miembro superior & 70 & 27 & 97 & 24.4 \\
\hline - Miembro inferior & 12 & 4 & 16 & 4 \\
\hline - Tórax/abdomen & 1 & 0 & 1 & 0.2 \\
\hline - No especificada & 19 & 7 & 26 & 6.5 \\
\hline Heridas únicas & 279 & 69 & 348 & 87.8 \\
\hline Heridas múltiples & 30 & 18 & 48 & 12.1 \\
\hline Edad media (años) & $16.8 \pm 18.2$ & $27.1 \pm 22.3$ & & \\
\hline Mediana de edad (años) & 11 & & & \\
\hline $\operatorname{Sexo}(M / F)$ & $183 / 126$ & $48 / 39$ & $231 / 165$ & $58 / 41$ \\
\hline$<180>18$ años & $217 / 92$ & $39 / 48$ & $256 / 140$ & $64 / 35$ \\
\hline Hospitalización & 2 & 4 & 6 & 1.5 \\
\hline $\begin{array}{l}\text { Grupo de edad: } \\
\text { - Lactantes: } 1 \text { mes-2 años y } 11 \text { meses }\end{array}$ & 38 & 4 & 42 & 10.6 \\
\hline - Preescolares: 3-6 años & 72 & 13 & 85 & 21.4 \\
\hline - Escolares: > 6-12 años & 77 & 13 & 90 & 22.7 \\
\hline - Adolescentes: 13-18 años & 30 & 9 & 39 & 9.8 \\
\hline - Adultos: > 18-50 años & 67 & 33 & 100 & 25.2 \\
\hline - Adultos mayores: > 50 años & 25 & 15 & 40 & 10.1 \\
\hline Total & 309 & 87 & 396 & $78 / 22$ \\
\hline
\end{tabular}

se observó en el grupo de adultos (18-50 años), con 109 casos $(26.2 \%)$, seguidos de los adultos mayores (>50 años) con 45 casos (10.8\%).

La localización anatómica más frecuente fue la región de la cara, con 255 casos (61.3\%), seguida de la zona de labio y nariz (24.8\%). De los 255 casos en la cara, 199 ocurrieron en menores de 18 años (78\%), y el grupo con más pacientes fue el de los preescolares, con 75 casos (29.4\%). Para la extremidad superior se registraron 109 casos (26.2\%), y de estos, la mano fue la localización anatómica más frecuente, con 84 casos (77.1\%) (Fig. 2). De los 109 casos, 82 ocurrieron en mayores de 18 años $(77.2 \%)$ y el grupo con más casos fue el de adultos, con 55 casos $(50.5 \%)$. Finalmente, en la extremidad inferior se registraron 18 casos (4.3\%), en la zona de cabeza y cuello hubo seis casos $(1.4 \%)$ y por último hubo un caso en la zona del tórax, que fueron las localizaciones anatómicas menos frecuentes.
La mayoría de las heridas se presentaron en una sola región anatómica, con heridas múltiples con 367 casos $(88.2 \%)$, y con menor frecuencia en más de dos regiones anatómicas, con 49 casos (11.8\%) (Tabla 1). La presentación como herida única no se observó en este estudio. Todos los casos requirieron intervención y la mayoría pudieron resolverse únicamente con cierre primario (que incluye aseo quirúrgico, irrigación, desbridación o remodelación de bordes, más antibioticoterapia profiláctica) y anestesia local en el área de urgencias, correspondiendo a 309 casos $(74.3 \%)$, y en menor proporción se requirieron además técnicas reconstructivas para su reparación, en 89 casos (21.4\%) (Tabla 1). En 12 casos (2.9\%) no hubo necesidad de cierre primario por las características de las lesiones (laceraciones superficiales y dermoabrasiones), en las cuales solo se realizó aseo quirúrgico y curación con ungüento de antibiótico, más esquema de antibióticos profilácticos. En 


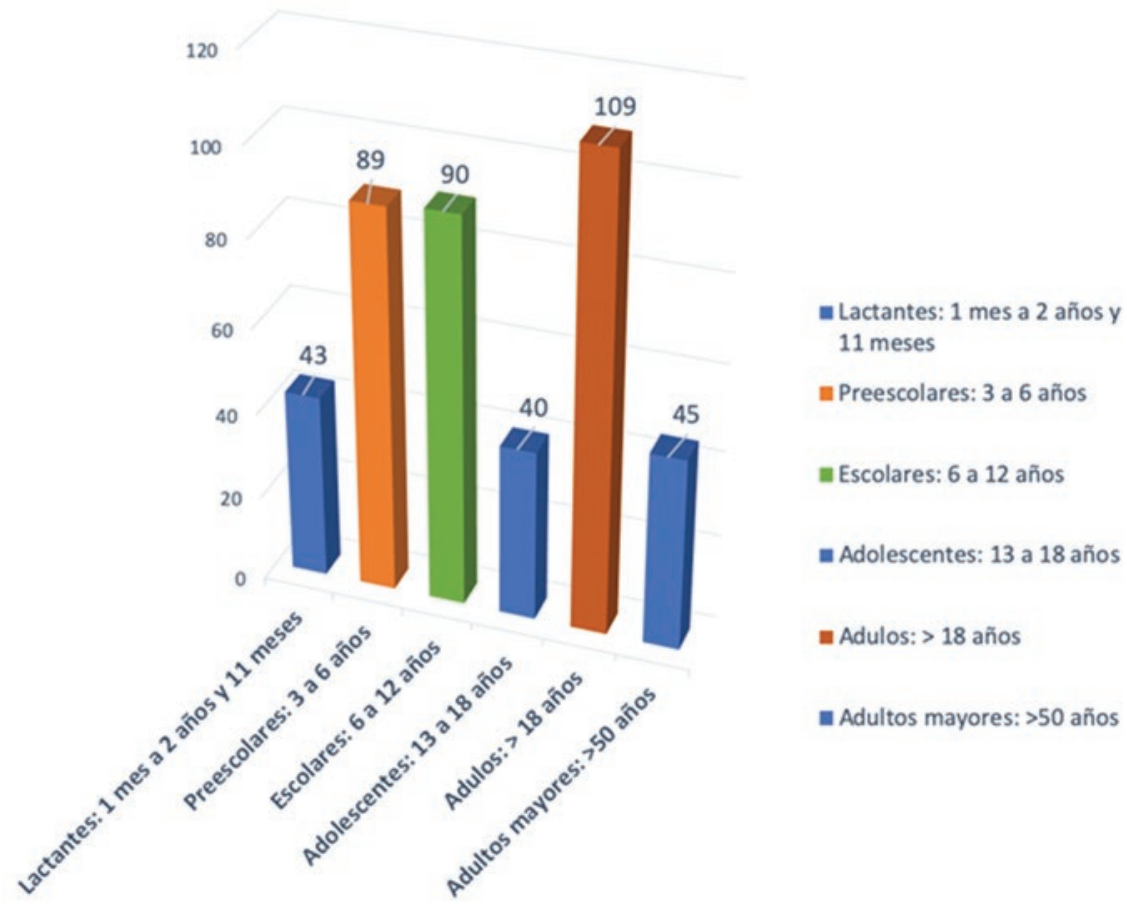

Figura 1. Grupos de edad.

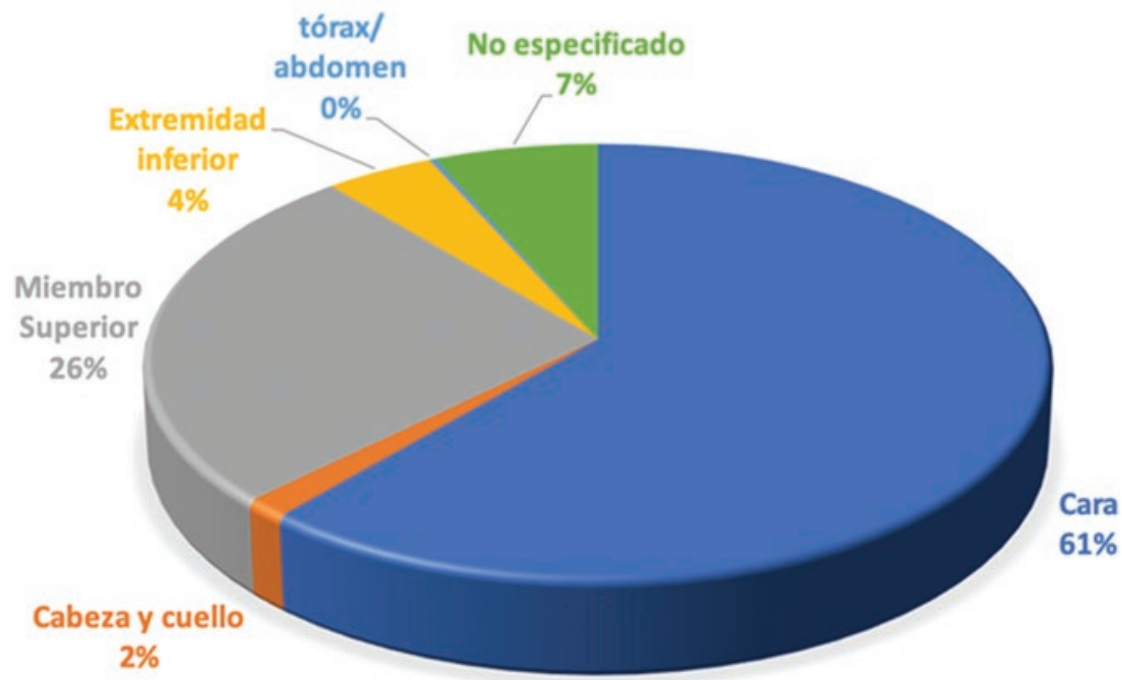

Figura 2. Localización anatómica.

seis casos (1.4\%) hubo necesidad de hospitalización por la gravedad de las heridas o infección ya establecida antes de su llegada a nuestro centro; de ellos, cuatro eran preescolares, debido a la gravedad y el número de lesiones que requirieron anestesia general para su reparación en la sala de operaciones. Los otros dos casos de hospitalización correspondieron al grupo de adultos debido a infección de la herida con datos de infección sistémica; ambos se presentaron con más de 24 horas de evolución, sin tratamiento de las heridas, y se manejaron con aseo quirúrgico, desbridación de bordes y colocación de drenajes más antibioticoterapia hasta la estabilización de la herida para el cierre definitivo. Respecto a la edad, en los mayores de 18 años fue más frecuente la necesidad de procedimientos reconstructivos. La edad promedio de los pacientes que pudieron atenderse solo con cierre primario fue 16.8 años, mientras que la edad promedio de los que requirieron además procesos reconstructivos fue de 27.3 años. 


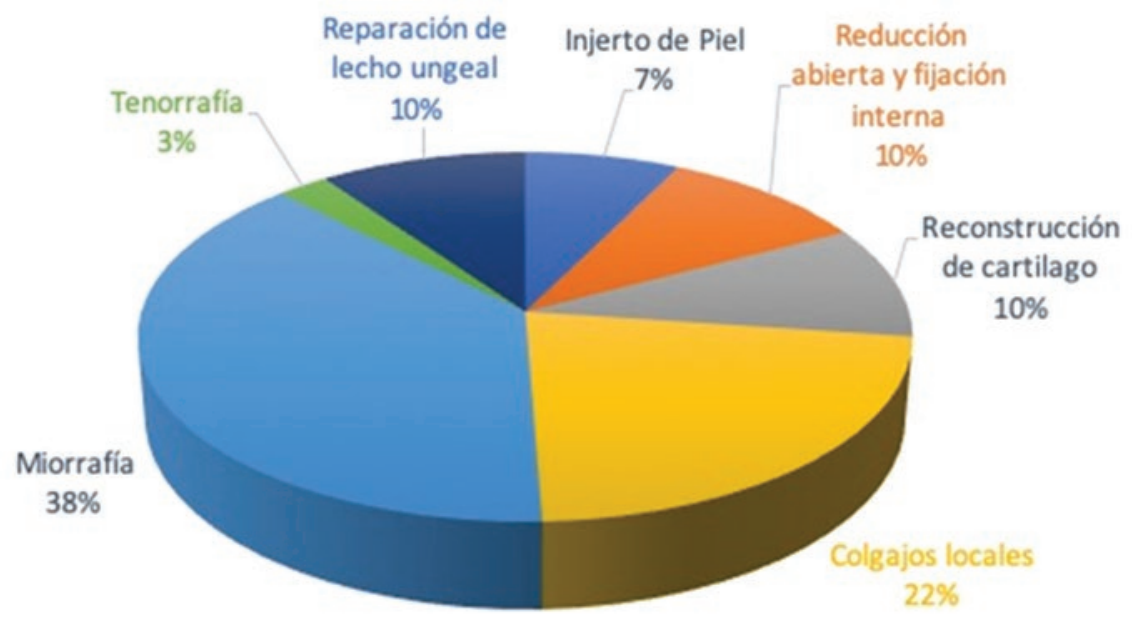

Figura 3. Procesos reconstructivos.

En cuanto a los procesos reconstructivos, el mayor número de casos ocurrió en la cara (54 de los 107; $50.4 \%)$, y la técnica más frecuente $(28.9 \%)$ fue el cierre primario más reparación muscular mediante miorrafia, seguida del avance de colgajos en sus diferentes variedades (16.8\%) (Fig. 3). Otro tipo de procesos reconstructivos que se utilizaron en menor proporción fueron reducción abierta y fijación interna de fracturas $(7.48 \%)$, reconstrucción de lecho ungueal $(7.5 \%)$ y reconstrucción de cartílago auricular (7.48\%).

Los pacientes que requirieron intervención en la sala de operaciones recibieron esquema intravenoso con ampicilina/sulbactam, y la mayoría de los que requirieron intervención en el servicio de urgencias recibieron esquema oral con amoxicilina-ácido clavulánico (875/125 mg dos veces al día, $60 \mathrm{mg} / \mathrm{kg}$ de amoxicilina por dosis, dos veces al día) más clindamicina por 7 días. En los alérgicos a penicilina se empleó un esquema basado en trimetoprima-sulfametoxazol (4-6 $\mathrm{mg} / \mathrm{kg}$ componente trimetoprima por dosis, dos veces al día) por 7 días. Todos los pacientes fueron revalorados a las 24 y las 72 horas hasta la resolución de la herida, con registros fotográficos. En todos los casos se administraron antibióticos profilácticos. En ocho casos (2\%) se reportaron datos de infección localizada tras el manejo con cierre primario (seis en un miembro superior y dos en un miembro inferior); todos los casos tenían más de 12 horas de evolución y fueron manejados con lavado de la herida cada 24 horas más esquema de antibiótico de amplio espectro con adecuada evolución; en ningún caso hubo necesidad de hospitalización. No se reportó mortalidad.

\section{Discusión}

Actualmente no existe un consenso establecido en México en cuanto al manejo de las mordeduras de perro de acuerdo con el tipo de herida, la gravedad, la localización anatómica y la edad, y por supuesto no existen aún estudios en México que describan el pronóstico funcional y social a largo plazo de estos pacientes debido a las secuelas con las que pudieran resultar. Uno de los objetivos principales de este trabajo es describir la experiencia en el manejo de heridas por mordedura de perro en uno de los centros más importantes de cirugía plástica y reconstructiva en México, y al mismo tiempo proponer un algoritmo de manejo estandarizado para todos los niveles de atención, con el fin de mejorar el pronóstico estético $y$ funcional de estos pacientes.

En México existen pocos artículos publicados acerca de mordeduras de perro, la mayoría con un enfoque epidemiológico ${ }^{2,3}$, y coinciden con la literatura internacional al describir que el grupo más afectado son los menores de 18 años, en especial los niños de 5-9 $a^{n}{ }^{2-4}$. En nuestro estudio, los menores de 18 años fueron los más vulnerables y el sexo masculino tuvo más predisposición que el femenino para sufrir mordeduras de perro. La mayoría de los casos se reportaron en el grupo de preescolares y escolares, que en suma conformaron la mayoría de los casos (Tabla 1). Concordando con la literatura, en nuestro estudio la cara fue el sitio con más frecuencia afectado (Fig. 2), presentándose clínicamente como múltiples heridas; cabe mencionar que es poco frecuente que las mordeduras de perro se presenten como una sola herida. Un gran número de pacientes presentaron heridas 


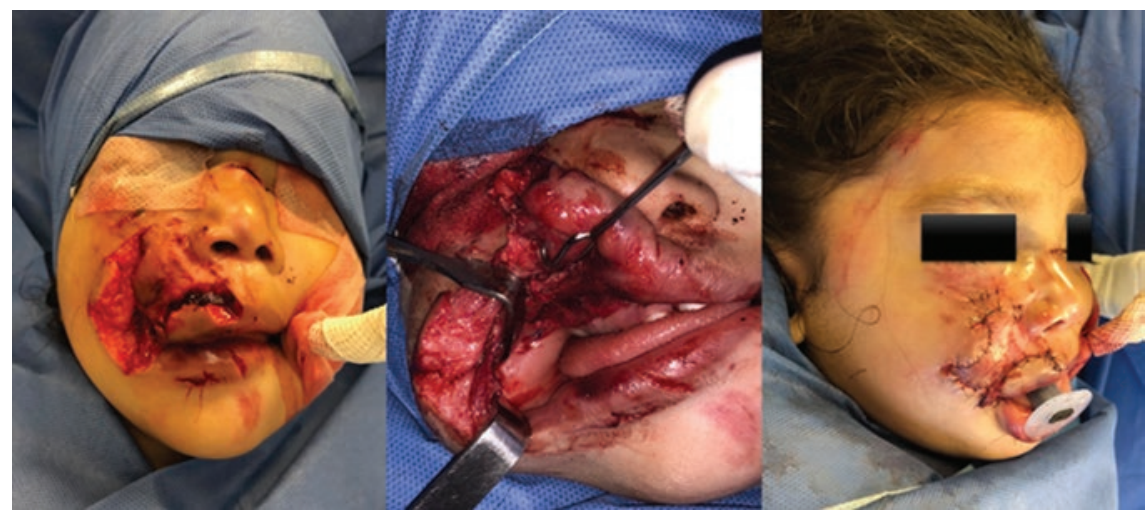

Figura 4. Caso representativo: niña de 3 años víctima de mordedura de perro de raza Pitbull de 6 horas de evolución, con lesión inicial en zonas III y IV de Seckel que involucra piel, tejido celular subcutáneo, músculo y pérdida de la comisura labial. Reconstrucción inmediata mediante ferulización del conducto de Stenon, comisuloplastia y cierre primario.

localizadas en la región de los labios y la nariz, los párpados y la región auricular, las cuales en su totalidad requirieron procesos reconstructivos (Fig. 3). La afectación de la cara en la infancia se explica por diversos factores, principalmente por la estatura del niño, que aproxima la cabeza al hocico del can, además del comportamiento desinhibido que el niño demuestra hacia el perro, como sorprenderlo mientras se encuentra comiendo, o el juego agresivo repetido por parte de los varones principalmente, así como la falta de desarrollo de habilidades motoras para protegerse la cara $^{3-6}$, lo cual predispone a que la gravedad de las lesiones sea mayor y como consecuencia los niños con heridas en esta región generalmente requieran atención en un hospital de segundo o tercer nivel (pediatría, cirugía plástica, oftalmología, otorrinolaringología $)^{7-10}$. Un factor importante que determina la gravedad de las lesiones es la raza del perro, aunque es difícil documentarla en todos los casos. En nuestra experiencia, en tres casos en los que se pudo verificar la raza, la variedad Pitbull se asoció con necesidad de procesos reconstructivos y mayor tiempo de intervención quirúrgica y estancia hospitalaria debido a la complejidad de las lesiones ${ }^{10,11}$, y aunque aún no puede establecerse una relación de la gravedad de las lesiones con esta raza debido a la falta de estudios en nuestro país, en diversos artículos realizados en los Estados Unidos se indica esta raza como una causa importante de la complejidad de las heridas e incluso de muerte en los pacientes ${ }^{12}$. Cabe mencionar que la fuerza en la mordida de esta raza es de aproximadamente 235 libras por pulgada cuadrada, y aunque razas como el Rottweiler (328 lb) y el Pastor Alemán $(238 \mathrm{lb})$ tienen mayor fuerza ${ }^{13}$, el patrón de ataque no interrumpido y la tenacidad con la que el Pitbull lo realiza resulta en una mayor gravedad que con cualquier otra raza ${ }^{14}$, sumando además el factor de que en su mayoría son perros entrenados para pelea. En estudios realizados en Denver (Colorado, EE.UU.) se evidenció que, a pesar de la legislación que existe en el Estado para la prohibición esta raza, aún continúa siendo la más involucrada en ataques catastróficos ${ }^{4,15}$.

En nuestro estudio ocurrieron cuatro casos de hospitalización debido a la gravedad de las heridas, tres en preescolares y uno en escolares. Ocurrieron en la cara y el miembro superior, respectivamente, y todos requirieron procedimientos reconstructivos en la sala de operaciones por parte del servicio de cirugía plástica (Fig. 4). A medida que el niño crece y en los mayores de 18 años las mordeduras son más frecuentes en el miembro superior, tal como se evidencia en nuestro estudio, en el que la mayoría de los adultos presentaron lesiones en este (sobre todo en la mano), explicado por la capacidad que tiene el adulto de defenderse con el miembro superior y evitar un ataque directo, y además de que un número considerable son a causa de separar a dos perros durante una pelea ${ }^{8-16}$ (Fig. 2).

Las mordeduras de perro deben considerarse como heridas contaminadas debido a la flora del hocico canino, en el cual existen más de 64 especies patógenas para el ser humano ${ }^{17}$. La infección que resulta de la mordedura generalmente es polimicrobiana; sin embargo, los patógenos más comunes en orden de prevalencia son Pasteurella, Staphylococcus y Streptococcus sp., y anaerobios como Bacteroides, Fusobacterium y Prevotella. La bacteria Pasteurella multocida se aísla en el $50 \%$ de las mordeduras de perro ${ }^{18}$. Capnocytophaga canimorsus es una bacteria rara, pero que puede causar bacteriemia y sepsis rápidamente, sobre todo en pacientes asplénicos, con 
enfermedades crónicas o bajo tratamiento inmunosupresor; aunque es sumamente rara, es importante considerarla cuando un paciente persiste con infección de la herida y sepsis a pesar de un tratamiento intensivo correcto ${ }^{19}$. Si bien el tiempo desde que ocurrió el ataque hasta el momento de la atención es uno de los principales factores para predecir si una herida tiene riesgo de infección, es un dato que no se registra en todos los casos. En nuestro estudio hubo dos casos con necesidad de hospitalización por infección de la herida más datos de infección sistémica; ambos ocurrieron en el grupo de adultos y se localizaron en el miembro superior, tratándose de heridas múltiples, de gravedad moderada y con más de 24 horas de evolución desde el ataque hasta el momento de su llegada al hospital.

\section{Algoritmo Gea González para el manejo de heridas por mordedura de perro}

En nuestra experiencia como centro de referencia en cirugía reconstructiva en el sur de la Ciudad de México, donde se recibe un gran número de pacientes con este diagnóstico derivados de otros centros de atención, generalmente por la gravedad de las lesiones o la región anatómica que se encuentra involucrada (mano y cara), tratamos las lesiones de acuerdo con la gravedad, para lo cual proponemos un algoritmo de manejo (Fig. 5).

Para laceraciones únicas superficiales o dermoabrasión sin pérdida de tejido ni solución de continuidad preferimos irrigar la zona con abundante agua o solución salina a presión media por 15 minutos para no dañar los tejidos, aplicar abundante jabón con clorhexidina en repetidas ocasiones y vendar la herida con ungüento de antibiótico, y citar al paciente a las 24 y 72 horas para vigilar la evolución, sobre todo en heridas localizadas en el miembro superior. Si se trata de múltiples dermoabrasiones puede agregarse un esquema de antibiótico oral profiláctico, y consulta de seguimiento en 24,48 y 72 horas vigilando la evolución de la herida ${ }^{7}$; sin embargo, los pacientes que se presentan con estas características en nuestra institución son muy pocos (14; $3.4 \%$ ).

Las heridas puntiformes únicas que no involucran la cara ni la mano pueden ser manejadas en un primer nivel de atención. Recomendamos irrigación profusa, desbridación de bordes no viables, aplicar ungüento con antibiótico y vendaje, realizar evaluación expectante de la herida cada 24 horas y, si existen datos clínicos locales o sistémicos de infección, referir a segundo nivel para manejo de herida infectada.

En heridas puntiformes múltiples o que involucren la cara o la mano se recomienda referir a segundo nivel con cirugía plástica. Realizamos una infiltración local con anestesia, posteriormente irrigación profusa por 15 minutos con agua o solución salina, lavado de heridas con clorhexidina, desbridación de bordes no viables, cierre primario, antibióticos profilácticos y vigilancia expectante cada 24 horas. Si existen datos clínicos de infección se realiza hospitalización, manejo de la herida infectada con antibióticos intravenosos, lavado cada 24 horas y sonda de irrigación continua o sistema de presión negativa (o ambos).

Para lesiones únicas o múltiples de gran tamaño que incluyan avulsiones complejas, pérdida de tejido, amputación, tejido triturado, afección de estructuras profundas (músculos, tendones, vasos y nervios), primordialmente en la cara y la mano, recomendamos siempre la valoración por los servicios de cirugía plástica y reconstructiva, cirugía pediátrica, oftalmología u otorrinolaringología en su caso, ya que un incorrecto manejo de las heridas en dichas regiones tiene como consecuencia múltiples complicaciones y secuelas (lesión del nervio facial, lesión del conducto lagrimal, lesión del conducto de Stenon, epifora, alopecia, cicatrices hipertróficas de mala calidad, pérdida de función de los músculos, isquemia, necrosis de la zona, infecciones y secuelas estéticas y psicológicas) ${ }^{20,21}$.Es importante contar siempre con imágenes radiológicas para las heridas en los miembros (radiografías simples) y con tomografía de cráneo y de macizo facial para las heridas localizadas en la cara, ya que no es infrecuente encontrar fracturas asociadas, sobre todo en ataques graves y de perros de raza mediana 0 grande $^{11-22}$. Preferimos el manejo inicial con irrigación profusa de la herida con solución salina por 15 minutos y posteriormente aplicación de anestesia local subdérmica con lidocaína simple (dosis de $4 \mathrm{mg} / \mathrm{kg}$ ) o lidocaína con epinefrina (dosis de $7 \mathrm{mg} / \mathrm{kg}$ ) para tener una mejor manipulación de la herida. Una vez anestesiada la herida, volvemos a irrigar por $15 \mathrm{mi}-$ nutos con solución salina y jabón con clorhexidina con el fin de eliminar restos de tierra, para posteriormente explorar la herida. Es importante identificar los bordes necróticos no viables, y de ser así, desbridarlos (ya que este enfoque reduce un $30 \%$ el riesgo de infección) ${ }^{23}$. Posteriormente se realiza una exploración quirúrgica detallada con el fin de buscar afección de estructuras profundas (nervio facial, conducto lagrimal, vasos, tendones, etc.) para evitar la pérdida de 


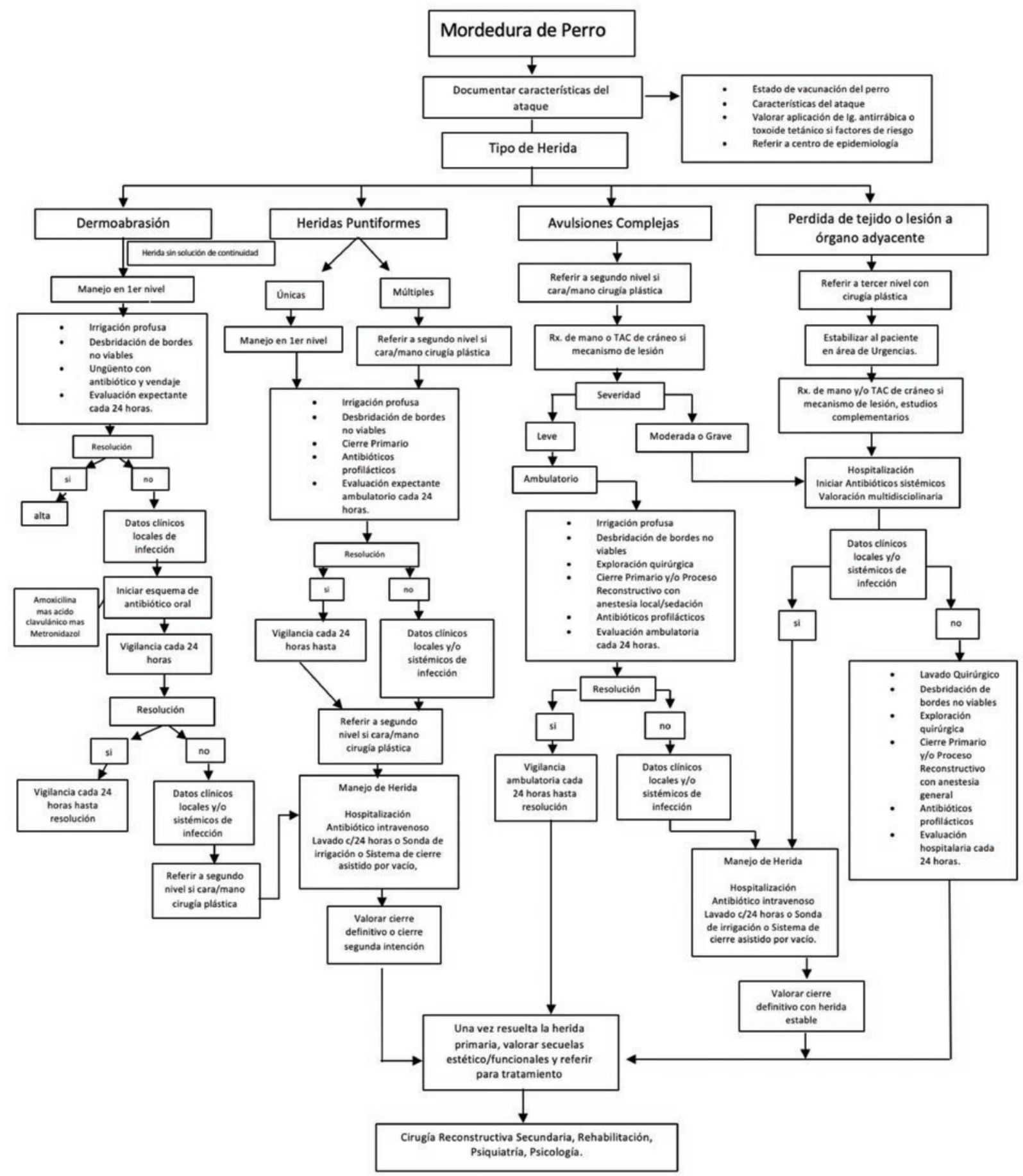

Figura 5. Algoritmo de tratamiento para el manejo de heridas por mordedura de perro.

la función, complicaciones o incluso isquemia y necrosis del área. Una vez finalizada la exploración puede procederse al cierre primario. Para estructuras profundas preferimos suturas absorbibles $\left(V_{i c r y}{ }^{\circledR}\right.$ 4-0, 5-0 o 6-0); de ser posible, utilizar la menor cantidad de suturas subcutáneas para no aumentar el riesgo de infección por cuerpo extraño 22 ; para los planos superficiales utilizamos monofilamento de nailon (4-0,
5-0, 6-0 ${ }^{24}$. Se pautan antibióticos profilácticos, así como consulta de seguimiento para evolución de la herida en 24,48 y 72 horas y una semana posterior si no hay complicaciones. En los pacientes que no toleran el manejo con anestesia local puede realizarse el cierre primario con sedación (p. ej., ketamina); esto ocurre con frecuencia en pacientes pediátricos, en quienes el umbral del dolor es muy bajo ${ }^{7-22}$. 


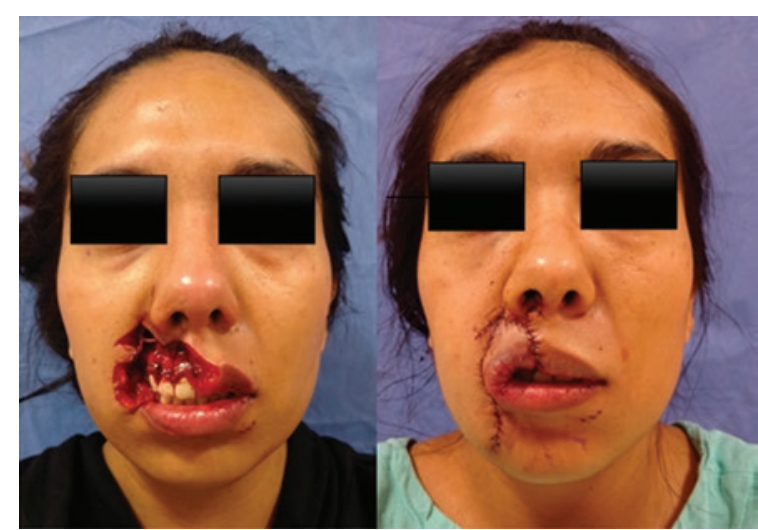

Figura 6. Caso representativo: mujer de 21 años con herida por mordedura de perro de espesor total que involucra cuatro subunidades estéticas: labio superior rojo y blanco, filtrum y arco de cupido, así como comisura labial derecha. Reconstrucción inmediata con colgajo Estlander.

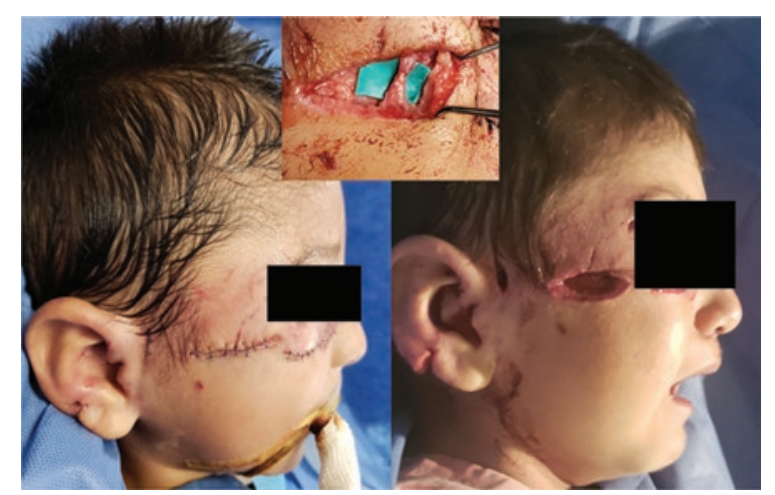

Figura 7. Caso representativo. Masculino de 2 años con multiples mordeduras de perro en nariz, mejilla, parpado superior e inferior derecho e inferior izquierdo, así como en región malar izquierda. Herida en Zona II y IV de Seckel con lesión parcial de la rama frontal del nervio facial y fractura orbitocigomatíca derecha y post reconstrucción inmediata con remodelación de bordes, cierre primario, neurorrafía y reducción abierta y fijación interna.

Cuando existen laceraciones importantes, avulsión completa de tejido, pérdida de tejido, fracturas asociadas y lesión de estructuras profundas (sistema nervioso central, globo ocular), el manejo interdisciplinario en un tercer nivel es primordial. Los procedimientos reconstructivos serán necesarios y el cirujano plástico evaluará la técnica de la escalera o elevador reconstructivo para reparar el defecto, en un primer o segundo tiempo. En nuestro estudio, los pacientes que requirieron procesos reconstructivos fueron menos frecuentes que los que requirieron solo cierre primario en el área de urgencias (74.3 vs. $25.7 \%)$. Las técnicas reconstructivas más empleadas en nuestro estudio fueron el cierre primario más la reparación muscular mediante miorrafia, avance de colgajos, toma y aplicación de injertos (más frecuentes de espesor parcial que de espesor total), reconstrucción de cartílago auricular (todos estos más prevalentes en la cara), y reducción abierta y fijación interna de fracturas más cierre primario para la mano (Figs. 6 y 7). De manera frecuente, cuando no es posible realizar el cierre primario nos apoyamos en el sistema de presión negativa para fijación de injertos, desinfectar la herida y prepararla para un proceso reconstructivo definitivo (colgajos microquirúrgicos). Es importante destacar que los pacientes que requieren un manejo reconstructivo deben ser seguidos periódicamente, debido a las secuelas funcionales y psicológicas que pudieran resultar, aunque muchas veces los pacientes abandonan el seguimiento. Actualmente contamos con 10 casos de secuelas que requerirán otras cirugías reconstructivas (expansores de tejidos, colgajos locales, colgajos libres, transferencias nerviosas, etc.) para poder atenuarlas y reincorporar al paciente a su vida social. Todas las lesiones fueron en la cara y se trata de secuelas estéticas en los labios, los ojos y los párpados, afección del nervio facial, cicatrices de mala calidad y alopecia.

Solo cuando las heridas son de complejidad moderada 0 tienen más de 24 horas de evolución, con datos clínicos locales o sistémicos de infección (leucocitosis $>12,000$, secreción purulenta, edema, hipertermia, hiperemia, herida dolorosa a la manipulación, fiebre y formación abscesos)22, recomendamos la hospitalización del paciente, realizar la desbridación y remodelación de bordes, y posteriormente colocar un drenaje o un sistema de presión negativa e iniciar esquema de antibioticoterapia intravenosa con revaloración cada 24 horas para definir el momento del cierre definitivo. En casos especiales puede valorarse el cierre por segunda intención?.

En México, el manejo de las heridas por mordedura de perro se establece de acuerdo con la NOM-011SSA2-2011 para la prevención y control de la rabia ${ }^{25}$ y la Guía para la atención médica y antirrábica de la persona expuesta al virus de la rabia ${ }^{26}$.Cabe mencionar que en la versión anterior de esta, de 1994, se recomendaba retrasar y no realizar el cierre primario en el primer contacto debido a la creencia de un aumento en el riesgo de infección, sobre todo en la cara, la mano y los genitales. En su versión más actual se menciona que el cierre primario puede realizarse cuando se requiera por fines estéticos en un segundo o tercer nivel, siempre acompañado de la aplicación de suero antirrábico si el factor de exposición es elevado (contacto con saliva en mucosas, heridas penetrantes, heridas en la cara, la mano o los genitales). 
Se recomienda también desbridar las heridas y afrontar con puntos simples; sin embargo, gracias al éxito de las campañas de vacunación en perros, así como la prevención con suero antirrábico en casos con alto riesgo, la incidencia actual de rabia en nuestro país es nula, lo cual favorece que se realice el cierre primario en el primer contacto.

Anteriormente se creía que el cierre primario aumentaba el riesgo de infección local y por virus de la rabia, y que tenía mayor riesgo de infección de la herida ${ }^{24-27}$, pero diversas series recientes han demostrado a través del tiempo que no existe una disminución del riesgo de infección cuando se retrasa el cierre primario, y que incluso aumenta el riesgo de esta, además de que el pronóstico estético y funcional es peor en los pacientes en los que se difiere la reparación de la herida ${ }^{28}$; por último, los costos del tratamiento aumentan al doble, ya que para realizar el manejo inicial con curaciones de la herida en la mayoría de los casos se requiere anestesia local o sedación, y en caso de retrasar el cierre se necesitarían múltiples consultas de seguimiento con necesidad de volver a anestesiar la herida para realizar las curaciones o el cierre primario, lo que implica mayor tiempo, más dinero, secuelas psicológicas en niños y dolor por manipulación constante para el paciente ${ }^{29-32}$.

En cuanto al manejo operativo, en todos los niveles de atención el abordaje inicial deberá ir acompañado de un adecuado lavado quirúrgico, como ha sido descrito anteriormente. Si se considera de inicio que la complejidad de la herida requiere manejo en un segundo o tercer nivel de atención, se recomienda lavar la herida superficialmente, eliminar el material externo que predisponga a infección, vendar la herida, iniciar antibiótico y referir a la institución más cercana. Para un segundo nivel se deberá iniciar un adecuado lavado quirúrgico con irrigación, desbridación y remodelación de bordes no viables, lo cual disminuye la incidencia de infección en un 30\% ${ }^{30-37}$. Este manejo terapéutico se ha llevado a cabo en nuestra institución por más de 30 años, con una tasa de infección baja para el número de pacientes que son atendidos, y este éxito radica en el seguimiento estricto de los pacientes, que se realiza con citas a las $24,48,72$ horas y hasta la resolución de la misma, para poder detectar y atender tempranamente infecciones localizadas o complicaciones, sobre todo en los miembros, donde la tasa de infección es más elevada en comparación con la cara, teniendo resultados estéticos y funcionales satisfactorios, y reincorporando tempranamente al paciente a su vida laboral y social ${ }^{22}$.
Otro punto controversial en cuanto al manejo de las mordeduras de perro es el uso de antibióticos profilácticos. En algunas series se ha reportado que no existe diferencia en la incidencia de infección entre los pacientes que reciben antibióticos y lo que no los reciben ${ }^{30-35}$.Dependiendo del estudio, la incidencia de infección después del manejo de mordeduras de perro puede variar del 1 al $14 \%^{29,31,36}$. Solo existen dos ensayos aleatorizados prospectivos que evaluaron la tasa de infección posterior al cierre primario en comparación con el cierre diferido en heridas de mordedura de perro, y no encontraron un mayor riesgo de infección debido a la sutura primaria de las heridas, lo que confirma informes similares en la literatura ${ }^{37,38}$. En nuestro estudio, la tasa de infección de la herida posterior al cierre primario es del $2 \%$. De acuerdo con nuestra experiencia, recomendamos siempre manejo con antibióticos profilácticos en heridas de gravedad moderada a alta localizadas en la cara y los miembros, sobre todo si hubo necesidad de tratamiento quirúrgico, y en heridas puntiformes, lo cual nos ha brindado excelentes resultados, con una tasa de infección baja. Recomendamos el esquema oral con amoxicilina-ácido clavulánico (875/125 mg dos veces al día, $22.5 \mathrm{mg} / \mathrm{kg}$ de amoxicilina por dosis, dos veces al día) más clindamicina por 7 días. En los alérgicos a la penicilina se puede indicar un esquema basado en trimetoprima-sulfametoxazol (4 a $6 \mathrm{mg} / \mathrm{kg}$ de trimetoprima por dosis, dos veces al día) por 7 días. En todos los casos que se presenten con el diagnóstico de mordedura de perro deberá recabarse una historia médica detallada que incluya antecedentes de importancia y estado de vacunación del paciente, referir al servicio correspondiente para realizar escrutinio de rabia y tétanos de acuerdo con los factores de riesgo, documentar las características del ataque (raza del perro, perro conocido, ataque provocado, estatus de vacunación del animal) y recabar las características de la herida con registros fotográficos antes y después de su reparación. Es importante establecer una adecuada relación entre médico y paciente para evitar el abandono del tratamiento.

Debido a la naturaleza retrospectiva del estudio, los datos de los registros médicos, ciertas variables como la raza del perro y las características del ataque no pudieron ser verificados en todos los casos. Al ser un centro de referencia para esta patología, el factor del tiempo desde que ocurrió el ataque hasta su llegada al hospital tampoco pudo verificarse en todos los registros, dato que es relevante para predecir el riesgo de infección. Por otro lado, la tasa de infección 
reportada en nuestro estudio se asumió por el control que se tiene a los 2, 3 y 7 días posteriores al tratamiento y hasta la resolución de la herida en todos los pacientes, encontrado una tasa baja de infección posterior al cierre primario. No se reportó mortalidad.

Realizar estudios prospectivos con seguimiento a largo plazo de los pacientes tendría más peso estadístico y ayudaría a conocer el porcentaje de secuelas funcionales y psicológicas, además del número de cirugías reconstructivas que son necesarias para reincorporar al paciente a una vida normal, así como los elevados costos. Es necesario realizar campañas hospitalarias que ayuden a mejorar el registro de las mordeduras de perro, así como establecer un algoritmo de atención en todos los eslabones del sistema de salud, así como también una campaña nacional para la prevención y la educación respecto a las mordeduras de perro, sobre todo enfocada a padres de familia que tienen perros en casa, lo cual pudiera tener un alto impacto en la reducción de la incidencia de las heridas por esta causa.

\section{Conclusiones}

Las heridas por mordedura de perro continúan siendo un problema de salud pública y representan una significativa porción de los motivos de consulta en los servicios de urgencias, sobre todo en la población pediátrica, en la que son más frecuente. Se deberá contar siempre con valoración por cirugía plástica en un segundo o tercer nivel de atención para heridas con complejidad moderada a grave, sobre todo en la cara y la mano. Proponer un algoritmo de manejo basado en la experiencia en un centro de referencia en cirugía plástica en todos los niveles de atención mejora el pronóstico de los pacientes y disminuye las complicaciones y las secuelas. El uso de colgajos en sus diferentes variedades, así como el uso de injertos, sigue siendo el caballo de batalla para el cirujano plástico; sin embargo, como se ha observado en este estudio, dependiendo de la gravedad de la herida se usarán otras armas del arsenal más complejas, como los colgajos microquirúrgicos, y de manera no infrecuente se requerirán cirugías secundarias para atenuar las secuelas. El cierre primario es seguro y tiene mejor resultado estético y funcional cuando se realiza un adecuado manejo de la herida (lavado quirúrgico profuso, desbridación de los bordes necróticos y antibióticos profilácticos), y deberá ser realizado siempre que sea posible. A menudo, la miorrafia (más frecuente del músculo orbicular de los labios y los ojos) o la tenorrafia en los miembros superiores acompañan al cierre primario. Es indispensable realizar el seguimiento a las 24,48 y 72 horas posteriores al tratamiento primario y siempre hasta la resolución de la herida, con el motivo de detectar y tratar oportunamente complicaciones como infecciones, las cuales ocurren en su mayoría en las extremidades y en pacientes adultos, en ocasiones con necesidad de manejo intrahospitalario. Actualmente, siguiendo la misma línea de investigación se están realizando estudios prospectivos para comparar diferentes técnicas reconstructivas, así como la eficacia de los antibióticos profilácticos y la tasa de infección posterior al cierre primario, para poder complementar esta primera fase descriptiva.

\section{Agradecimientos}

Agradecemos a cada uno de los autores por el empeño dedicado para la realización de este trabajo, así como al cuerpo del servicio de cirugía plástica y reconstructiva, que en su desempeño diario se dedican al manejo óptimo y al bienestar de los pacientes.

\section{Conflicto de intereses}

Los autores de este trabajo no tenemos interés financiero para declarar en relación con el contenido de este artículo. La financiación corrió a cuenta de los mismos autores.

\section{Responsabilidades éticas}

Protección de personas y animales. Los autores declaran que para esta investigación no se han realizado experimentos en seres humanos ni en animales.

Confidencialidad de los datos. Los autores declaran que han seguido los protocolos de su centro de trabajo sobre la publicación de datos de pacientes.

Derecho a la privacidad y consentimiento informado. Los autores han obtenido el consentimiento informado de los pacientes y/o sujetos referidos en el artículo. Este documento obra en poder del autor de correspondencia.

\section{Bibliografía}

1. American Society of Plastic Surgeons. 2014 Plastic surgery statistic report. Disponible en: www.plasticsurgery.org

2. Hernández Arroyo DA. Mordedura de perro: enfoque epidemiológico de las lesiones causadas por mordedura de perro. Revista de Enfermedades Infecciosas en Pediatría. 2009;23:13-20.

3. Velázquez Reyes V, Flores Ávila G, Pedroso Balandrano A. Treatment of craniofacial region wounds caused by dog bite. Revista Odontológica Mexicana. 2013;17:243-50 


\section{E. Chávez-Serna, et al.: Manejo de heridas por mordedura de perro en un hospital de tercer nivel}

4. Chen HH, Neumeier AT, Davies BW, Durairaj VD. Analysis of pediatric facial dog bites. Craniomaxillofac Trauma Reconstr. 2013;6:225-32.

5. Baeza-Herrera C, González- Mateos T, Castillo-Aguirre Al, Godoy-Esquivel $\mathrm{AH}$, Maraboto Millan JA. Mordedura craneofacial por perro. Acta Pediatr Mex. 2010;31:89-94.

6. Schalamon J, Ainoedhofer H, Singer G, Petnehazy T, Mayr J, Kiss K, et al. Analysis of dog bites in children who are younger than 17 years. Pediatrics. 2006;117:e374

7. Alizadeh K, Shayesteh A, Li Xu M. An algorithmic approach to operative management of complex pediatric dog bites: 3-year review of a level regional referral pediatric trauma hospital. Plast Reconstr Surg Glob Open. 2017:5:e1431.

8. Mannion CJ, Graham A. Dog bite injuries in hospital practice. Br J Hosp Med. 2016;77:C165-8

9. Kaye AE, Belz JM, Kirschner RE. Pediatric dog bite injuries: a 5-year review of the experience at the Children's Hospital of Philadelphia. Plast Reconstr Surg. 2009;124):551-8.

10. O'Brien DC, Andre TB, Robinson AD, Squires LD, Tollefson TT. Dog bites of the head and neck: an evaluation of a common pediatric trauma and associated treatment. Am J Otolaryngol. 2015;36:32-8.

11. Garvey EM, Twitchell DK, Ragar R, Egan JC, Jamshidi R. Morbidity of pediatric dog bites: a case series at a level one pediatric trauma center. J Pediatr Surg. 2015;50:343-6.

12. Bini JK, Cohn SM, Acosta SM, McFarland MJ, Muir MT, Michalek JE; TRISAT Clinical Trials Group. Mortality, mauling, and maiming by vicious dogs. Ann Surg. 2011;253:791-7.

13. Barr DB. Dangerous encounters: bite force. Disponible en: http://natgeotv.com/asia/webisodes/web-shows/dangerous-encounters?videold $=11922377$

14. Loewe CL, Diaz FJ, Bechinski J. Pit bull mauling deaths in Detroit. Am J Forensic Med Pathol. 2007;28:356-60.

15. Gurunluoglu R, Glasgow M, Arton J, Bronsert M. Retrospective analysis of facial dog bite injuries at a level I trauma center in the Denver metro area. J Trauma Acute Care Surg. 2014;76:1294-300.

16. Méndez MRG, Gómez MT, Somoza IA, Liras JM, Pais PE, Vela DN Mordedura de perro. Análisis de 654 casos en 10 años. An Esp Pediatr. 2002;56:425-9.

17. Talan DA, Citron DM, Abrahamian FM, Moran GJ, Goldstein EJ. Bacteriologic analysis of infected dog and cat bites. Emergency Medicine Animal Bite Infection Study Group. N Engl J Med. 1999;340:85-92.

18. Goldstein EJ. New horizons in the bacteriology, antimicrobial susceptibility and therapy of animal bite wounds. J Med Microbiol. 1998;47:95-7.

19. Meyers B, Schoeman JP, Goddard A, Picard J. The bacteriology and antimicrobial susceptibility of infected and non-infected dog bite wounds: fifty cases. Vet Microbiol. 2008;127:360-8.
20. Hallock G. Dog bites of the face with tissue loss. J Craniomaxillofac Trauma. 1996;2:49-55.

21. Gurunluoglu R, Glasgow M, Arton J, Bronsert M. Retrospective analysis of facial dog bite injuries at a level I trauma center in the Denver metro area. J Trauma Acute Care Surg. 2014;76:1294-300.

22. Lin W, Patil PM. Facial dog attack injuries. Indian J Surg. 2015;77:55-8.

23. Akhtar N, Smith MJ, McKirdy S, Page RE. Surgical delay in the management of dog bite injuries in children, does it increase the risk of infection? J Plast Reconstruct Aesthet Surg. 2006;59:80-5.

24. Pinsolle J, Phan E, Coustal B, Manciet P, Courtois I. [Dog bites on the face. Apropos of 200 cases]. Ann Chir Plast Esthet. 1993:38:452-6.

25. Secretaría de Salud. Modificación a la Norma Oficial Mexicana NOM011-SSA1993 Para la Prevención y Control de la Rabia. Diario Oficial de la Federación, 23 de junio de 2000.

26. Secretaría de Salud. Subsecretaria de Prevención y Promoción a la Salud. Guía para la atención médica y antirrábica de la persona expuesta al virus de la rabia. CENAPRECE; octubre de 2018.

27. Jones RC, Shires GT. Bites and stings of animals and insects. En: Schwartz SI, Shires GT, Spencer FC, Storer EH, editors. Principles of surgery. New York: McGraw-Hill; 1979. p. 232-42.

28. Wu PS, Beres A, Tashjian DB, Moriarty KP. Primary repair of facial dog bite injuries in children. Pediatr Emerg Care. 2011:27:801-3.

29. Mcheik JN, Vergnes $P$, Bondonny JM. Treatment of facial dog bite injuries in children: a retrospective study. J Pediatr Surg. 2000;35:580-3.

30. Lackmann G, Wolfgang D, Isselstein G, Tollner U. Surgical treatment of facial dog bites injuries in children. J Craniomaxillofac Surg. 1992;20:81-6.

31. Monroy A, Behar P, Nagy M, Poje C, Pizzuto M, Brodsky L. Head and neck dog bites in children. Otolaryngol Head Neck Surg. 2009; 140:354-7.

32. Kesting MR, Holzle F, Pox C, Thurmuller P, Wolff KD. Animal bite injuries to the head: 132 cases. Br J Oral Maxillofac Surg. 2006;44:235-9.

33. Callham ML. Treatment of common dog bites: infection risk factors. JACEP. 1978;7:83-7.

34. Kuvat SV, Bozkurt M, Kapi E, Karakol P, Yaçsar Z, Güven E. Our treatment approaches in head-neck injuries caused by animal bites. J Craniofac Surg. 2011;22:1507-10.

35. Morgan JP, Haug HR, Murphy MT. Management of facial dog bite injuries. J Oral Maxillofac Surg. 1995;53:435-41.

36. Mitchell RB, Nañez G, Wagner JD, et al. Dog bites of the scalp, face, and neck in children. Laryngoscope. 2003:113:492-5.

37. Paschos NK, Makris EA, Gantsos A, Georgoulis AD. Primary closure versus non-closure of dog bite wounds. a randomised controlled trial. Injury. 2014;45:237-40.

38. Maimaris C, Quinton DN. Dog-bite lacerations: a controlled trial of primary wound closure. Arch Emerg Med. 1988;5:156-61. 